ESAIM: PROCEEDINGS, October 2007, Vol. 22, 15-20

Gabriel Caloz \& Monique Dauge, Editors

\title{
OPTIMAL TRANSPORTATION OF FLOWS AND NONLINEAR ELECTROMAGNETISM*
}

\author{
YANN BRENIER ${ }^{1}$
}

Dedicated to Michel Crouzeix

\begin{abstract}
The concept of optimal transportation of densities has become very popular in the 10 last years with numerous applications in very different fields of pure and applied mathematics. (See [14] for a recent review.) In this contribution, we discuss a generalization of this concept for flows (and not only densities) and its connection with the nonlinear Electromagnetism of Born and Infeld [4].
\end{abstract}

\section{INTERPOLATION OF FLUID MOTIONS}

For various purposes (including interpolation of moving pictures, but not only), we are interested in interpolating two given "fluid motions" in a smooth bounded domain $D$ in the plane $R^{2}$ (we do not consider in the present paper the case of a general Riemannian manifold -such as a sphere- and concentrate on the euclidean case with two space dimensions only). Each of the two fluid motions can be described by a flow, i.e. a time-dependent family of orientation preserving diffeomorphisms of $D$, respectively denoted by

$$
a \in D \rightarrow \Phi^{-}(t, a) \in D
$$

and

$$
a \in D \rightarrow \Phi^{+}(t, a) \in D
$$

Here $t$ denotes the time variable (valued in a given fixed interval $[0, T]$ ) and $a$ a reference position in $D$ for each fluid particle. A convenient normalization is:

$$
\Phi^{-}(0, a)=\Phi^{+}(0, a)=a .
$$

The corresponding 'Eulerian' density and velocity fields of each fluid are implicitly defined, in a standard fashion, by:

$$
\begin{gathered}
v^{-}\left(t, \Phi^{-}(t, a)\right)=\partial_{t} \Phi^{-}(t, a) \in R^{2}, \quad v^{+}\left(t, \Phi^{+}(t, a)\right)=\partial_{t} \Phi^{+}(t, a) \in R^{2}, \\
\rho^{-}(t, \Phi(t, a)) \frac{\partial\left(\Phi_{1}^{-}(t, a), \Phi_{2}^{-}(t, a)\right)}{\partial\left(a_{1}, a_{2}\right)}=\rho_{0}^{-}(a),
\end{gathered}
$$

\footnotetext{
* CNRS, Université de Nice

1 FR2800 W. Doeblin, Mathématiques,

Parc Valrose, 06108 Nice Cedex 02
} 


$$
\rho^{+}(t, \Phi(t, a)) \frac{\partial\left(\Phi_{1}^{+}(t, a), \Phi_{2}^{+}(t, a)\right)}{\partial\left(a_{1}, a_{2}\right)}=\rho_{0}^{+}(a),
$$

where $\rho_{0}^{-}$and $\rho_{0}^{+}$denote the density at time $t=0$ of both fluids.

We want to interpolate, in some optimal fashion to be defined later, these flows by a family of flows denoted by

$$
(t, a, s) \in[0, T] \times D \times[0,1] \rightarrow \Phi(t, a, s) \in D
$$

where $s$ stands for the interpolation parameter valued in $[0,1]$ so that:

$$
\Phi(t, a, 0)=\Phi^{-}(t, a), \quad \Phi(t, a, 1)=\Phi^{+}(t, a), \quad \Phi(0, a, s)=a .
$$

So we are looking for a two parameter $(t$ and $s$ ) family of diffeomorphisms of $D$. Three 'Eulerian fields' on $D$ can be associated with $\Phi$.

Using notation $x:=\left(x_{1}, x_{2}, x_{3}:=s\right) \in Q:=D \times[0,1]$, these fields $\rho(t, x), v(t, x), b(t, x)$ are implicitly defined for each $t \in[0, T]$ by:

$$
\begin{gathered}
v(t, \Phi(t, a, s), s)=\left(\partial_{t} \Phi_{1}(t, a, s), \partial_{t} \Phi_{2}(t, a, s)\right) \in R^{2}, \\
b(t, \Phi(t, a, s), s)=\left(\partial_{s} \Phi_{1}(t, a, s), \partial_{s} \Phi_{2}(t, a, s)\right) \in R^{2}, \\
\rho(t, \Phi(t, a, s), s) \frac{\partial\left(\Phi_{1}(t, a, s), \Phi_{2}(t, a, s)\right)}{\partial\left(a_{1}, a_{2}\right)}=\rho_{0}(a, s),
\end{gathered}
$$

where the initial density $\rho_{0}$ has to be defined. These three fields satisfy:

$$
\begin{gathered}
\partial_{t} \rho+\partial_{1}\left(\rho v_{1}\right)+\partial_{2}\left(\rho v_{2}\right)=0 \\
\partial_{1}\left(\rho b_{1}\right)+\partial_{2}\left(\rho b_{2}\right)+\partial_{3} \rho=0 \\
\partial_{t} b_{1}+v_{1} \partial_{1} b_{1}+v_{2} \partial_{2} b_{1}=b_{1} \partial_{1} v_{1}+b_{2} \partial_{2} v_{1}+\partial_{3} v_{1}, \\
\partial_{t} b_{2}+v_{1} \partial_{1} b_{2}+v_{2} \partial_{2} b_{2}=b_{1} \partial_{1} v_{2}+b_{2} \partial_{2} v_{2}+\partial_{3} v_{2} .
\end{gathered}
$$

Notice that the two last equations just express (in Eulerian form) the symmetry property $\partial_{s t}^{2} \Phi=\partial_{t s}^{2} \Phi$ and can be equivalently written, in conservation form:

$$
\begin{aligned}
& \partial_{t}\left(\rho b_{1}\right)+\partial_{2}\left(\rho b_{1} v_{2}\right)=\partial_{2}\left(\rho b_{2} v_{1}\right)+\partial_{3}\left(\rho v_{1}\right), \\
& \partial_{t}\left(\rho b_{2}\right)+\partial_{1}\left(\rho b_{2} v_{1}\right)=\partial_{1}\left(\rho b_{1} v_{2}\right)+\partial_{3}\left(\rho v_{2}\right) .
\end{aligned}
$$

Concerning boundary conditions, we see that, at $x_{3}=0$ and $x_{3}=1$, the fields $\rho$ and $v$ are prescribed. Along $[0, T] \times \partial D \times[0,1]$, by construction, the fields $v$ and $b$ are parallel to the boundary. (More precisely $\rho v \cdot n=\rho b \cdot n=0$, where $n$ denotes the outward normal to $D$ and $\cdot$ the inner product in $R^{2}$.) At $t=0$, the normalization $\Phi(t=0, a, s)=a, \forall a \in D$, implies $b=0$. At $t=T$, there is no natural boundary condition.

\section{The STATIC APPROACH: ClASSICAL OPTIMAL TRANSPORT OF DENSITIES}

In the static approach, we forget the dynamical part of the problem, considering $t$ just as a parameter and ignoring $v$. Thus, dropping $t$ in our notations, we are just looking for $(\rho(x), b(x), x \in Q=D \times[0,1])$ such that:

$$
\partial_{1}\left(\rho b_{1}\right)+\partial_{2}\left(\rho b_{2}\right)+\partial_{3} \rho=0,
$$

inside $Q$, with prescribed values of $\rho(x)$ at $x_{3}=0$ and $x_{3}=1$, and $b$ parallel to $\partial D$. So far, we have a widely underdetermined problem. To single out interesting solutions, we introduce optimality criteria such as

$$
I(\rho, b):=\int_{Q} \frac{\rho(x)^{\alpha}|b(x)|^{\beta}}{\beta} d x,
$$


for some fixed exponents $\alpha, \beta \geq 1$, and look for minimizers $(\rho, b)$.

For $\alpha=1, \beta=2$, we recover the "quadratic" transportation problem (in its "Benamou-Brenier" formulation $[1,3]$ ), which is linked to several important PDEs (Monge-Ampère, Hamilton-Jacobi and Euler) [5] and has numerous applications (see [14] for a rather comprehensive review).

The choice $\alpha=\beta=1$ leads to the original Monge problem [13] "sur les déblais et les remblais", which is fairly degenerate and mathematically delicate [1].

Finally, $\alpha=\beta=2$ corresponds to the Moser construction [12], as explained in [6]. All three problems are convex minimization problems, not in $(\rho, b)$ but rather in $(\rho, \rho b)$. They can be written as a saddle point problem:

$$
\begin{aligned}
& \inf _{\rho, b} \sup _{\theta} \int_{Q}\left\{\frac{\rho^{\alpha}|b|^{\beta}}{\beta}-\partial_{3} \theta \rho-\partial_{1} \theta \rho b_{1}-\partial_{2} \theta \rho b_{2}\right\} d x_{1} d x_{2} d x_{3} \\
+ & \int_{D}\left\{\theta\left(x_{1}, x_{2}, 1\right) \rho\left(x_{1}, x_{2}, 1\right)-\theta\left(x_{1}, x_{2}, 0\right) \rho\left(x_{1}, x_{2}, 0\right)\right\} d x_{1} d x_{2},
\end{aligned}
$$

where $(\theta(x), x \in D)$ is the Lagrangian multiplier attached to constraint:

$$
\partial_{1}\left(\rho b_{1}\right)+\partial_{2}\left(\rho b_{2}\right)+\partial_{3} \rho=0 .
$$

The resulting optimality equation are quite different depending on $\alpha$ and $\beta$.

Let us first consider the case $\alpha=1, \beta=2$, when the domain $D$ is convex. The optimality equations are

$$
\begin{gathered}
b(x)=\left(\partial_{1} \theta(x), \partial_{2} \theta(x)\right), \\
\partial_{3} \theta+\frac{\left(\partial_{1} \theta\right)^{2}+\left(\partial_{2} \theta\right)^{2}}{2}=0,
\end{gathered}
$$

which means that the Lagrange multiplier $\theta$ solves a quadratic Hamilton-Jacobi equation. Notice that, along the boundaries $x_{3}=0$ and $x_{3}=1, \rho$, subject to

$$
\partial_{1}\left(\rho b_{1}\right)+\partial_{2}\left(\rho b_{2}\right)+\partial_{3} \rho=0
$$

is prescribed but not $\theta$ ! Thus, there is no straightforward method, directly based on the resolution of the Hamilton-Jacobi equation, to solve the resulting optimal transportation problem.

Let us now consider the Moser case $\alpha=\beta=2$. The optimality conditions are:

$$
\partial_{3} \theta=0, \quad \rho b=\left(\partial_{1} \theta, \partial_{2} \theta\right)
$$

where $\theta$ now depends only on $\left(x_{1}, x_{2}\right)$. Thus,

$$
\partial_{1}\left(\rho b_{1}\right)+\partial_{2}\left(\rho b_{2}\right)+\partial_{3} \rho=0
$$

has two consequences. First, $\partial_{3} \rho$ does not depend on $x_{3}$, which means

$$
\rho(x)=\rho\left(x_{1}, x_{2}, 1\right) x_{3}+\rho\left(x_{1}, x_{2}, 0\right)\left(1-x_{3}\right) .
$$

Second, $\theta$ solves:

$$
\rho\left(x_{1}, x_{2}, 1\right)-\rho\left(x_{1}, x_{2}, 0\right)+\Delta \theta\left(x_{1}, x_{2}\right)=0,
$$

inside $D$, with homogeneous Neumann boundary conditions along $\partial D$. So, we see that $\rho$ is trivially obtained by linear interpolation and $b$ is well defined from $\theta$, provided the given densities at $x_{3}=0$ and $x_{3}=1$ are strictly 
positive on $D$.

Then, the recovery of the Moser interpolating flow $(\Phi(a, s), s \in[0,1], a \in D)$ is just a matter of integrating in $s=x_{3} \in[0,1]$ the ODE:

for each $a \in D$.

$$
\partial_{s} \Phi(a, s)=b(\Phi(a, s), s), \quad \Phi(0, a)=a,
$$

\section{The dynamical approach: EleCtromagnetic notations}

Let us go back to the full dynamical setting. More compact formulae can be obtained by introducing electromagnetic notations [7] as follows. Let $B(t, x) \in R^{3}, E(t, x) \in R^{3}$ be defined for $t \in[0, T]$ and $x \in D \times[0,1]$ by:

$$
\begin{gathered}
B=\left(\rho b_{1}, \rho b_{2}, \rho\right), \\
E=\left(-\rho v_{2}, \rho v_{1},\left(b_{1} v_{2}-b_{2} v_{1}\right) \rho\right) .
\end{gathered}
$$

Then, the differential constraints obtained for the fields $(\rho, v, b)$ simply mean, in terms of $(B, E)$ :

$$
\nabla \cdot B=0, \quad \partial_{t} B+\nabla \times E=0,
$$

where $\nabla=\left(\partial_{1}, \partial_{2}, \partial_{3}\right)$ and $\cdot$ is the inner product in $R^{3}$. So $B$ and $E$ both encode the interpolation process and behave as an electromagnetic field linked by the homogeneous part of the standard Maxwell equations. Notice, however, that $B$ and $E$ are linked by an additional algebraic constraint:

$$
E \cdot B=0
$$

to be satisfied pointwise, which is not standard in classical Electromagnetism. Let us now look at the boundary condition satisfied by $E, B$ along the boundary of $[0, T] \times D \times[0,1]$. Along the boundaries $x_{3}=0$ and $x_{3}=1$, $\rho, v_{1}, v_{2}$ are given to us. This exactly means that $B \cdot n$ and $E \times n$ are prescribed, with $n=(0,0,1)$. Along $t=0$, we have $\Phi(t=0, a, s)=a$, which implies $b_{1}=b_{2}=0$, and $\rho(t=0, a, s)=\rho_{0}(a, s)$. Thus $B$ is naturally prescribed at $t=0$. In both cases, these boundary conditions are natural in the framework of classical Electromagnetism.

\section{Optimal transportation OF FLOWS}

As suggested in an earlier work [7], a natural functional to be considered, in view of a variational principle for optimal transportation, is:

$$
\mathbf{A}[\Phi]:=\int_{D} A(\Phi(\cdot, a, \cdot)) d a,
$$

where $\Phi$ is an interpolation flow and $A(\Phi(\cdot, a, \cdot))$ is the area, for each fixed $a$, of the $2 \mathrm{D}$ surface

$$
\Phi(\cdot, a, \cdot): \quad(t, s) \in[0, T] \times[0,1] \rightarrow(t, \Phi(t, a, s), s) \in[0, T] \times D \times[0,1],
$$

in the ambient 4D space $R \times R^{2} \times R=R^{4}$. When $R^{4}$ is equipped with the Lorentzian metric $(-1,1,1,1)$ (keeping $t$ as time and considering $(a, s)$ as a three dimensional space variable), then the area is given by:

$$
A(\Phi(\cdot, a, \cdot)):=\int_{0}^{T} d t \int_{0}^{1} d s \sqrt{\left(1+\partial_{s} \Phi^{2}\right)\left(1-\partial_{t} \Phi^{2}\right)+\left(\partial_{t} \Phi \cdot \partial_{s} \Phi\right)^{2}} .
$$

If $R^{4}$ is equipped with the standard euclidean metric, the area becomes:

$$
A(\Phi(\cdot, a, \cdot)):=\int_{0}^{T} d t \int_{0}^{1} d s \sqrt{\left(1+\partial_{s} \Phi^{2}\right)\left(1+\partial_{t} \Phi^{2}\right)-\left(\partial_{t} \Phi \cdot \partial_{s} \Phi\right)^{2}} .
$$


The functional can be easily expressed in terms of the Eulerian fields $(\rho, v, b)$ as:

$$
\mathbf{A}[\Phi]=\int_{[0, T] \times D \times[0,1]} \rho \sqrt{\left(1+b^{2}\right)\left(1+\eta v^{2}\right)-\eta(v \cdot b)^{2}} d t d x,
$$

with $\eta=-1$ in the Lorentzian case and $\eta=1$ in the Euclidean case. Even better, in terms of the 'electromagnetic' field $(B, E)$, we simply have:

$$
\mathbf{A}[\Phi]=\int_{[0, T] \times D \times[0,1]} \sqrt{B^{2}+\eta E^{2}} d t d x,
$$

Thus, we have obtained a concept of optimal transportation for flows, in terms of a non-linear electromagnetic variational problem:

Find critical points $(E, B)$ of the functional

$$
\int_{[0, T] \times D \times[0,1]} \sqrt{B^{2}+\eta E^{2}} d t d x
$$

subject to the 'electromagnetic constraint'

$$
\nabla \cdot B=0, \quad \partial_{t} B+\nabla \times E=0,
$$

together with the pointwise algebraic constraint:

$$
E \cdot B=0
$$

\section{The Born-Infeld Electromagnetism}

Surprisingly enough, the algebraic constraint $E \cdot B=0$ is naturally penalized by substituting for the optimal transportation functional (4) the Electromagnetic functional introduced by Born and Infeld in 1934 [4]

$$
\mathbf{L}_{r}(E, B):=\int_{[0, T] \times D \times[0,1]} \sqrt{r^{2}+B^{2}+\eta E^{2}+(B \cdot E)^{2} \eta r^{-2}} d t d x
$$

where $r$ is a scaling parameter. In the limit $r \rightarrow \infty$, the BI model approaches the classical Maxwell functional, in the following sense:

$$
\lim _{r \rightarrow \infty} r\left(\mathbf{L}_{r}(E, B)-r\right)=\int_{[0, T] \times D \times[0,1]} \frac{1}{2}\left(B^{2}+\eta E^{2}\right) d t d x .
$$

In the limit $r \rightarrow 0$, the BI model approaches the optimal transportation model discussed in the previous section. (Geometrically and physically speaking, since extremal surfaces can be physically interpreted as classical relativistic strings, we are really looking for a foliation of the classical 4D Minkowski space by a continuum of strings.) Notice that the BI model was introduced in 1934 by Born and Infeld as a nonlinear cutoff theory to substitute for Maxwell's electromagnetism (which allows infinite electrostatic field for point charged particles). Here $r$ stands for an absolute bound for any electrostatic field (just as the speed of light is an absolute bound for any velocity in special relativity). Consistently, Maxwell's linear theory is recovered in the "low energy" limit $r \rightarrow \infty$. In the opposite "high energy" limit, as $r \rightarrow 0, r$ becomes a penalty parameter for the algebraic constraint $E \cdot B=0$. The high energy asymptotic analysis of the Born-Infeld model has been recently handled in collaboration with W.A. Yong in [10]. More generally, the study of the Born-Infeld model leads to many side issues. In particular, because of concentration and oscillation phenomena, the concept of extremal surface (i.e. relativistic strings) can be relaxed (subrelativistic strings and/or sticky strings) in different ways, as discussed in $[8,9]$. 


\section{Open mathematical issues}

In the Euclidean case $\eta=1$, it is natural to look for minimizers $(E, B)$ of $(4)$ subject to $(1,2)$ and appropriate boundary conditions. Because of the differential constraints, namely (1), the algebraic expression $E \cdot B$ can be written in divergence form and is weakly continuous in $(E, B)$ with respect to the weak topology of $L^{2}$. Unfortunately, we expect minimizing sequences to converge only as vector valued measures. So, it is unclear what is left from the algebraic constraint $E \cdot B=0$ under such a limiting process. A delicate interplay between concentration and oscillation effects may play a crucial role. So, we have obtained a challenging "supercritical compensated compactness" problem. Let us just observe that the algebraic constraint cannot be entirely relaxed. Indeed, an easy calculation shows that the convex hull of

$$
\int \sqrt{B^{2}+E^{2}} d x d t
$$

with constraint $E \cdot B=0$, is

$$
\int \sqrt{B^{2}+E^{2}+2|E \cdot B|} d x d t
$$

Also notice that various simplified versions of the problem can be addressed. If the $t$ dependence is given up, for instance, we may write (at least locally) $E$ as a gradient $E=\nabla \phi$, and we can renormalize the algebraic constraint as $\nabla \cdot(\psi B)=0$, where

$$
\psi=\frac{\phi}{\sqrt{1+\phi^{2}}}
$$

Then the problem becomes "critical", with the pairing of a vector valued measure $(B)$ and a bounded Borel function $(\psi)$. If, in addition, one space variable is dropped, the problem becomes explicitely integrable (and somewhat similar to a simple "disocclusion problem" [2] in image processing).

\section{REFERENCES}

[1] L. Ambrosio, N. Gigli, G. Savaré, Gradient flows in metric spaces and in the space of probability measures, Lectures in Mathematics ETH Zürich. Birkhuser Verlag, Basel, 2005.

[2] L. Ambrosio, S. Masnou, A direct variational approach to a problem arising in image reconstruction, Interfaces Free Bound. 5 (2003) 63-81.

[3] J.-D. Benamou, Y. Brenier, A Computational Fluid Mechanics solution to the Monge-Kantorovich mass transfer problem, Numer. Math. 84 (2000) 375-393.

[4] M. Born, L. Infeld, Foundations of the new field theory, Proc. Roy. Soc. London A 144 (1934) 425-451.

[5] Y.Brenier, Polar factorization and monotone rearrangement of vector-valued functions, Comm. Pure Appl. Math. 64 (1991) 375-417.

[6] Y.Brenier, Extended Monge-Kantorovich theory, Optimal transportation and applications, 91-121, Lecture Notes in Math., 1813, Springer, Berlin, 2003.

[7] Y. Brenier, A note on deformations of 2D fluid motions using 3D Born-Infeld equations, Monatsh. Math. 42 (2004) $113-122$.

[8] Y. Brenier, Order preserving vibrating strings and applications to electrodynamics and magnetohydrodynamics, Methods Appl. Anal. 1 (2004) 515-532.

[9] Y. Brenier, Non relativistic strings may be approximated by relativistic strings, Methods Appl. Anal. 12 (2005), no. 2, 153-167.

[10] Y. Brenier, W.A. Yong, Derivation of particle, string, and membrane motions from the Born-Infeld electromagnetism, J. Math. Phys. 6 (2005) 062305.

[11] Y. Brenier, Le système de Born-Infeld élargi: des ondes aux particules et aux cordes, Séminaire Equations aux dérivées partielles 2004-2005, Ecole Polytechnique (2005).

[12] B. Dacorogna, J. Moser, On a partial differential equation involving the Jacobian determinant, Ann. Inst. H. Poincaré Anal. Non Linéaire $\mathbf{7}(1990)$ 1-26.

[13] L.V. Kantorovich, On a problem of Monge, Uspekhi Mat. Nauk. 3 (1948), 225-226.

[14] C. Villani, Topics in optimal transportation, Graduate Studies in Mathematics, 58 AMS, Providence, RI, 2003. 